\title{
Recursive prestack depth migration using CFP gathers
}

\author{
Jan Thorbecke ${ }^{1}$ and A. J. Berkhout ${ }^{2}$
}

\begin{abstract}
The common-focus-point technology (CFP) describes prestack migration by focusing in two steps: emission and detection. The output of the first focusing step represents a CFP gather. This gather defines a shot record that represents the subsurface response resulting from a focused source wavefield. We propose applying the recursive shot-record, depthmigration algorithm to the CFP gathers of a seismic data volume and refer to this process as CFP-gather migration. In the situation of complex geology and/or low signal-to-noise ratio, CFP-based image gathers are easier to interpret for nonalignment than the conventional image gathers. This makes the CFP-based image gathers better suited for velocity analysis. This important property is illustrated by examples on the Marmousi model.
\end{abstract}

\section{INTRODUCTION}

Common-focus-point (CFP) gathers have been introduced by Berkhout $(1992,1997)$ and been further developed in the DELPHI project of the University of Delft (Thorbecke, 1997). CFP technology has been used successfully for velocity-independent redatuming (Kelamis et al., 1999), to estimate Green's functions and velocity models (Kabir and Verschuur, 1997; Bolte et al., 1999; Hegge et al., 1999; Brisbourne et al., 2000), and to suppress internal multiples (Berkhout and Verschuur, 2000). In this paper, CFP technology is used for an alternative approach to shot-record migration.

A CFP gather can be considered as a shot record, the source of which is given by its focusing operator. Hence, a CFP gather can be migrated by a generalized shot-record migration algorithm, where the source wavefield is given by the focusing operator and the shot record by the CFP gather. We first set up a general framework for shot-record migration and then integrate the CFP gathers into this framework.

\section{WRW MODEL}

The so-called WRW model (Berkout, 1980) is an attractive starting point for the derivation of new seismic processing algorithms. In this model, wavefields are formulated in the $(x, y, \omega)$ domain in terms of operators. In the discrete version, these operators represent vectors and matrices. Looking at primary wavefields only, we may write the following:

downgoing wavefield:

$$
\mathbf{S}_{j}^{+}\left(z_{m}, z_{s}\right)=\mathbf{W}\left(z_{m}, z_{s}\right) \mathbf{S}_{j}^{+}\left(z_{s}\right)
$$

upgoing wavefields:

$$
\Delta \mathbf{P}_{j}^{-}\left(z_{r}, z_{s}\right)=\sum_{m=1}^{M} \mathbf{W}\left(z_{r}, z_{m}\right) \mathbf{R}\left(z_{m}, z_{m}\right) \mathbf{S}_{j}^{+}\left(z_{m}, z_{s}\right) ;
$$

measurements:

$$
\Delta \mathbf{P}_{j}\left(z_{r}, z_{s}\right)=\mathbf{D}_{j}\left(z_{r}, z_{r}\right) \Delta \mathbf{P}_{j}^{-}\left(z_{r}, z_{s}\right)
$$

In the primary wavefield (equations $1 \mathrm{a}-\mathrm{c}$ ), detector-matrix $\mathbf{D}_{j}$ $\left(z_{r}, z_{r}\right)$ and source-vector $\mathbf{S}_{j}^{+}\left(z_{s}\right)$ represent the angle-dependent acquisition information for a one-shot record (geometry, field arrays, source signature), $j$ being the position of the source array. Matrices $\mathbf{W}\left(z_{r}, z_{m}\right)$ and $\mathbf{W}\left(z_{m}, z_{s}\right)$ quantify the angle-dependent propagation properties between all individual grid points of the acquisition surfaces $z_{r}$ and $z_{s}$ and depth level $z_{m}$, respectively (each row represents an upward- and downward-propagation operator, respectively). Ma$\operatorname{trix} \mathbf{R}\left(z_{m}, z_{m}\right)$ quantifies the angle-dependent reflection properties for downward-traveling waves (each column represents one reflection operator) at depth level $z_{m}$ (see Figure 1). For a complex overburden, the columns of $\mathbf{W}$ can define multiarrival events. The $\Delta$ before $\mathbf{P}_{j}$ in equation $1 \mathrm{~b}$ indicates reflection data without surface and internal multiples.

From the primary-wavefield equations $1 \mathrm{a}-\mathrm{c}$, it follows that the primary reflection measurements may also be written as (Figure 2):

$$
\Delta \mathbf{P}_{j}\left(z_{r}, z_{s}\right)=\mathbf{D}_{j}\left(z_{r}, z_{r}\right) \Delta \mathbf{X}\left(z_{r}, z_{s}\right) \mathbf{S}_{j}^{+}\left(z_{s}\right),
$$

Manuscript received by the Editor October 19, 2005; revised manuscript received May 18, 2006; published online November 3, 2006.

${ }_{1}^{1}$ Cray, Lambarenestraat 39, 2622 DN Delft, The Netherlands. E-mail: janth@ Xs4all.nl.

${ }^{2}$ Delft University of Technology, Faculty of Technology, Policy and Management, P. O. Box 5015, 2600 GA Delft, The Netherlands. E-mail: a.j.berkhout@ tudelft.nl.

(C) 2006 Society of Exploration Geophysicists. All rights reserved. 
where matrix $\Delta \mathbf{X}\left(z_{r}, z_{s}\right)$ defines the earth's multidimensional transfer operators for primary reflections, each column representing one transfer operator:

$$
\Delta \mathbf{X}\left(z_{r}, z_{s}\right)=\sum_{m=1}^{M} \mathbf{W}\left(z_{r}, z_{m}\right) \mathbf{R}\left(z_{m}, z_{m}\right) \mathbf{W}\left(z_{m}, z_{s}\right)
$$

Equations $2 \mathrm{a}$ and $\mathrm{b}$ are generally referred to as the WRW model (Berkhout, 1980). The WRW model presents a detail-hiding formulation of wavefield measurements that include the influence of acquisition arrays as well as mode conversion.

\section{GENERALIZED SHOT-RECORD MIGRATION}

The goal of migration is to remove the propagation effects of the primary wavefield and image the reflections at the correct positions in the subsurface. The generalized shot-record migration algorithm (Berkhout, 1982, p. 218-220) is given by

$$
\begin{aligned}
& \mathbf{S}_{j}^{+}\left(z_{m}, z_{s}\right)=\mathbf{W}\left(z_{m}, z_{s}\right) \mathbf{S}_{j}^{+}\left(z_{s}\right), \\
& \mathbf{P}_{j}^{-}\left(z_{m}, z_{s}\right)=\mathbf{F}\left(z_{m}, z_{r}\right) \mathbf{P}_{j}^{-}\left(z_{r}, z_{s}\right),
\end{aligned}
$$

where equation $3 \mathrm{a}$ represents forward extrapolation of the source wavefield and equation $3 \mathrm{~b}$ represents inverse extrapolation of the recorded wavefield. Note that $\mathbf{P}_{j}$ contains all internal and free surface multiples. The extrapolated wavefields are related to the reflection information at depth level $z_{m}$ by

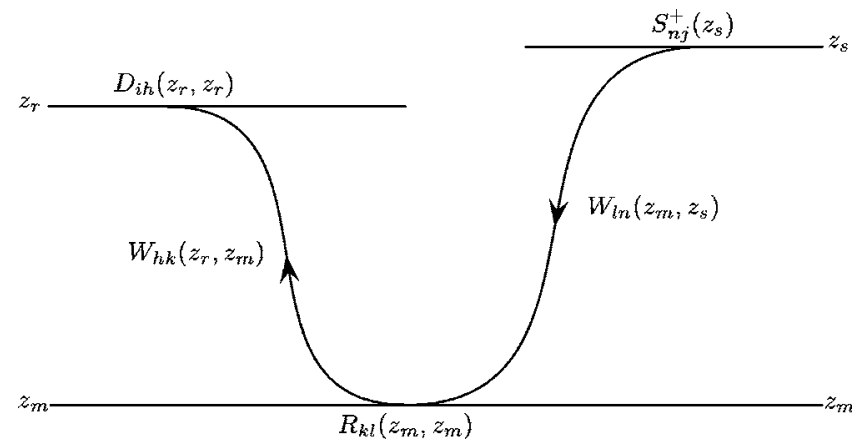

Figure 1. One basic element of the WRW model, visualized by a raypath. In the continuous formulation, the propagation and reflection operators are integral kernels. In the discrete formulation, they represent matrices. Note that depth levels $z_{s}, z_{r}$ and $z_{m}$ may be generalized to $z_{s}(x, y), z_{r}(x, y)$ and $z_{m}(x, y)$.

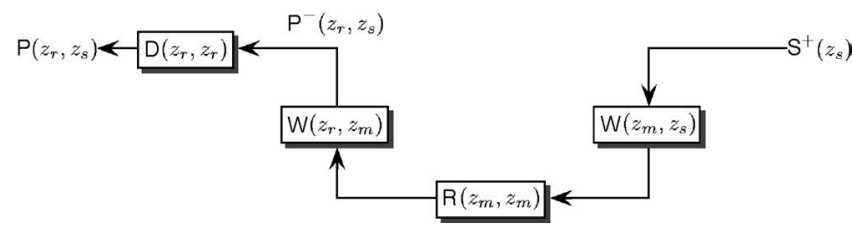

Figure 2. WRW forward model for primary reflections, representing the inverse imaging equations for seismic data. Note that in practice $z_{r}$ and $z_{s}$ often represent the acquisition surface $z_{0}$, meaning that $\mathbf{S}^{+}$ and $\mathbf{D}$ include the effect of the stress-free property of earth surface $z_{0}$.

$$
\mathbf{P}_{j}^{-}\left(z_{m}, z_{s}\right)=\mathbf{R}\left(z_{m}, z_{m}\right) \mathbf{S}_{j}^{+}\left(z_{m}, z_{s}\right)
$$

From this equation, the reflection information $\mathbf{R}\left(z_{m}, z_{m}\right)$ can be derived from $\mathbf{S}_{j}^{+}\left(z_{m}, z_{s}\right)$ and $\mathbf{P}_{j}^{-}\left(z_{m}, z_{s}\right)$ by matrix inversion.

Accurate extrapolation of wavefields is a computationally expensive task. By cleverly choosing the two wavefields $\mathbf{S}_{j}^{+}$and $\mathbf{P}_{j}^{-}$, it is possible to do the shot-record migration more efficiently. Let us first consider the recursive version of the shot-record migration equations $3 \mathrm{a}$ and $\mathrm{b}$.

\section{Recursive extrapolation for shot-record migration}

The forward and inverse extrapolation matrices represented by $\mathbf{W}$ and $\mathbf{F}$ in migration equations $3 \mathrm{a}$ and $\mathrm{b}$, respectively, can be implemented in many different ways. To handle the propagation through a complex subsurface, a recursive extrapolation scheme in the spacefrequency domain is used (Berkhout, 1982, p. 201-206):

$$
\begin{aligned}
\mathbf{W}\left(z_{m}, z_{s}\right) & =\mathbf{W}\left(z_{m}, z_{m-1}\right) \mathbf{W}\left(z_{m-1}, z_{m-2}\right) \ldots \mathbf{W}\left(z_{1}, z_{s}\right), \\
\mathbf{F}\left(z_{m}, z_{r}\right) & =\mathbf{F}\left(z_{m}, z_{m-1}\right) \mathbf{F}\left(z_{m-1}, z_{m-2}\right) \ldots \mathbf{F}\left(z_{1}, z_{r}\right),
\end{aligned}
$$

where for every recursive inverse operator, we use the approximation $\mathbf{F}=\left[\mathbf{W}^{*}\right]^{T}$. The rows of the recursive $\mathbf{W}$ and $\mathbf{F}$ matrices contain spatial convolution operators. These optimized finite-difference operators are calculated only once for the velocity and frequency range of interest and are stored in a table. During the extrapolation, the operator needed for the current frequency and velocity at position $\left(\boldsymbol{x}_{i}, z_{m}\right)$ is read from the table (Blacquière, 1989). For instance, one extrapolation step from $z_{m-1}$ to $z_{m}$ is given by the space-variant convolutions:

$$
\begin{aligned}
& \mathbf{S}_{j}^{+}\left(z_{m}, z_{s}\right)=\mathbf{W}\left(z_{m}, z_{m-1}\right) \mathbf{S}_{j}^{+}\left(z_{m-1}, z_{s}\right) \\
& \mathbf{P}_{j}^{-}\left(z_{m}, z_{s}\right)=\mathbf{F}\left(z_{m}, z_{m-1}\right) \mathbf{P}_{j}^{-}\left(z_{m-1}, z_{s}\right)
\end{aligned}
$$

where $\mathbf{W}$ and $\mathbf{F}$ are band matrices. For a detailed discussion about calculating short optimized extrapolation operators for $2 \mathrm{D}$ and $3 \mathrm{D}$ wavefield extrapolation, see Holberg (1988) and Thorbecke et al. (2004).

\section{Imaging principle for shot-record migration}

The reflection equation 4 shows that the reflectivity matrix $\mathbf{R}\left(z_{m}, z_{m}\right)$ cannot be determined from one shot record unless we assume that $\mathbf{R}\left(z_{m}, z_{m}\right)$ can be approximated by a diagonal matrix $\hat{\mathbf{R}}\left(z_{m}, z_{m}\right)$ of effective reflection coefficients. Effective reflection coefficients equal physical reflection coefficients if the reflection property at $z_{m}$ is largely angle independent or the incident wavefield is locally close to a plane wave, or both. Using the concept of effective reflection coefficients, we avoid full matrix inversion, and the reflection equation 4 may be rewritten as

$$
\mathbf{P}_{j}^{-}\left(z_{m}, z_{s}\right)=\hat{\mathbf{R}}\left(z_{m}, z_{m}\right) \mathbf{S}_{j}^{+}\left(z_{m}, z_{s}\right)
$$

for each temporal-frequency component. If we aim at frequencyaveraged reflection coefficients, stable inversion of imaging equation $7 \mathrm{a}$ is given by 


$$
\hat{\mathbf{R}}_{i j}\left(z_{m}, z_{m}\right)=\frac{\sum_{\omega} \mathbf{P}_{i j}^{-}\left(z_{m}, z_{s}\right)\left[\mathbf{S}_{i j}^{+}\left(z_{m}, z_{s}\right)\right]^{*}}{\sum_{\omega}\left|\mathbf{S}_{i j}^{+}\left(z_{m}, z_{s}\right)\right|^{2}},
$$

with $i$ ranging along $z_{m}$ and $j$ ranging along $z_{s}$.

In addition, if we also aim at one angle-averaged reflection coefficient for each grid point $\left(\mathbf{x}_{i}, z_{m}\right)$, another summation can be carried out:

$$
\hat{R}\left(\mathbf{x}_{i}, z_{m}\right)=\sum_{j} \frac{\sum_{\omega} \mathbf{P}_{i j}^{-}\left(z_{m}, z_{s}\right)\left[\mathbf{S}_{i j}^{+}\left(z_{m}, z_{s}\right)\right]^{*}}{\sum_{\omega}\left|\mathbf{S}_{i j}^{+}\left(z_{m}, z_{s}\right)\right|^{2}},
$$

with the summation over $j$ meaning a summation over all involved shot records.

For a given $i$, imaging equation $7 \mathrm{~b}$ shows the images of the involved shot records $\left(j\right.$ is variable) of grid point $\left(\mathbf{x}_{i}, z_{m}\right)$. If the underlying velocity distribution is correct, these images are aligned and can be found at $t=0$. If the process of extrapolation and imaging is applied for all depth levels of interest, the resulting images can be collected in an image gather for lateral position $\mathbf{x}_{i}$ (source position $\mathbf{x}_{j}$ and depth position $z_{m}$ are variable).

\section{Recursive migration of CFP gathers}

Migration of CFP gathers is similar to migration of shot records. Considering focusing in emission, a trace in a CFP gather is computed by weighted summation (in phase and amplitude) along the sources in a common-receiver gather so that the constructed wavefront $\mathbf{P}_{j}^{-}\left(z_{r}, z_{f}\right)$ originates from a notional source at point $\left(x_{j}, z_{f}\right)$ in the subsurface. The CFP gather for focusing in emission is given by

$$
\mathbf{P}_{j}^{-}\left(z_{r}, z_{f}\right)=\Delta \mathbf{P}\left(z_{r}, z_{s}\right) \mathbf{F}_{j}\left(z_{s}, z_{f}\right),
$$

where $\mathbf{F}_{j}\left(z_{s}, z_{f}\right)$ is the focusing operator containing the weights in amplitude and phase. Considering the WRW model, the CFP gather is a shot record with a focusing source array given by

$$
\mathbf{S}_{j}^{+}\left(z_{s}, z_{f}\right)=\mathbf{S}\left(z_{s}\right) \mathbf{F}_{j}\left(z_{s}, z_{f}\right) .
$$

The principle of combining shot gathers at the surface for the synthesis of areal source responses, also referred to as areal shot-record technology, was introduced by Berkhout (1992) and further investigated by Rietveld (1995). Extrapolation of the CFP gather in equation 8 and the focusing-source array in equation 9 from depth level $z_{m-1}$ to depth level $z_{m}$ can be represented by the recursive extrapolation equations $6 \mathrm{a}$ and $6 \mathrm{~b}$ with $z_{s}$ replaced by $z_{f}$ :

$$
\begin{aligned}
& \mathbf{S}_{j}^{+}\left(z_{m}, z_{f}\right)=\mathbf{W}\left(z_{m}, z_{m-1}\right) \mathbf{S}_{j}^{+}\left(z_{m-1}, z_{f}\right), \\
& \mathbf{P}_{j}^{-}\left(z_{m}, z_{f}\right)=\mathbf{F}\left(z_{m}, z_{m-1}\right) \mathbf{P}_{j}^{-}\left(z_{m-1}, z_{f}\right),
\end{aligned}
$$

To obtain the frequency-averaged reflection coefficients, we can apply the same steps as we have for the imaging principle for shotrecord migration, which leads to

$$
\hat{\mathbf{R}}_{i j}\left(z_{m}, z_{m}\right)=\frac{\sum_{\omega} \mathbf{P}_{i j}^{-}\left(z_{m}, z_{f}\right)\left[\mathbf{S}_{i j}^{+}\left(z_{m}, z_{f}\right)\right]^{*}}{\sum_{\omega}\left|\mathbf{S}_{i j}^{+}\left(z_{m}, z_{f}\right)\right|^{2}},
$$

with $i$ ranging along $z_{m}$ and $j$ now ranging along the level where focusing occurs, i.e., $z=z_{f}$. The source and receiver components in imaging equation 11 are given by extrapolation equations $10 \mathrm{a}$ and $\mathrm{b}$.
Imaging equation 11 contains source energy from all available shot records, which illuminate the focal point, while imaging equation $7 \mathrm{~b}$ contains energy from one shot only. Therefore, imaging equation 11 is significantly more stable than imaging equation $7 \mathrm{~b}$, particularly around the focal point.

The summation over shot records $j$ in imaging equation $7 \mathrm{c}$ adds more angle information of the same reflector and illuminates some extra parts because of the differences in source and receiver coordinates. Summation over migrated CFP gathers in imaging equation 11 adds more complete imaging results at different focal points $\left(\mathbf{x}_{j}, z_{f}\right)$ :

$$
\hat{R}\left(\mathbf{x}_{i}, z_{m}\right)=\sum_{j} \frac{\sum_{\omega} \mathbf{P}_{i j}^{-}\left(z_{m}, z_{f}\right)\left[\mathbf{S}_{i j}^{+}\left(z_{m}, z_{f}\right)\right]^{*}}{\sum_{\omega}\left|\mathbf{S}_{i j}^{+}\left(z_{m}, z_{f}\right)\right|^{2}} .
$$

If one can choose a smaller number of focal positions than the number of shot records with the same image quality, a more efficient migration can be carried out.

The construction of a CFP gather from shot records is explained by using numerical data based on the model shown in Figure $3 \mathrm{a}$. The numerical data are modeled with a fixed acquisition spread where the source positions are defined at every receiver position (201 shot positions with $\Delta x=15 \mathrm{~m}$ ). For the forward modeling of the data, an acoustic finite-difference algorithm is used.

The synthesis process for a focusing receiver with a focal point defined at the synclinal interface at $x=0$ and $z=950 \mathrm{~m}$ is detailed in Figure 3 (the focal point is indicated with a black bullet in Figure $3 \mathrm{a})$. The time-reversed focusing operator for the defined focus is shown in Figure 3b. This operator is applied to all common-shot gathers available. Three different common-shot gathers with source positions at $x=-495, x=0$, and $x=495 \mathrm{~m}$ are shown in Figure $3 \mathrm{c}-\mathrm{e}$, respectively. Trace convolution along the time axis in the shot gathers, with the traces in the synthesis operator, gives the intermediate synthesis results in Figure $3 \mathrm{f}-\mathrm{h}$. Note that in these intermediate synthesis results the bow tie of the syncline interface is still present. Summation over all traces in these results defines one trace of the CFP gather (Figure 3i). The most important contribution in the integrated result is determined by the Fresnel zone related to the focal point. If the focusing operator is correct, then the operator time at the source position is identical with the event time in the CFP trace. The summed trace is placed in the CFP gather at the source position. By carrying out the convolution and integration along the traces in the gather for all shot gathers available, the CFP gather for focusing in detection is constructed.

The events that are present in the shot record are also present in the intermediate synthesis result in Figure $3 \mathrm{f}-\mathrm{h}$. In Figure $3 \mathrm{f}$, four events are observed. The top event originates from the first reflector and can be regarded as a noncausal event with respect to the focal point. The event containing the triplication originates from the syncline boundary; the weak S-shaped event (indicated with (4)) originates from a diffraction point (positioned at $x=-750, z=100 \mathrm{~m}$ ); and the last event originates from the deepest boundary. In the CFP gather shown in Figure 3i, the reflection from the syncline (indicated by an arrow) and the deeper boundary are also visible. Because of the phase shift, the response of the first reflector is present in the CFP gather at negative times. The complex bow-tie response of the second reflector inthe shot record (Figure 3d) has been reduced to a simple hyperbolic event in the CFP gather.

Based on a collection of CFP gathers, a CFP-offset section can beconstructed by selecting a fixed offset from the focal point from ev- 


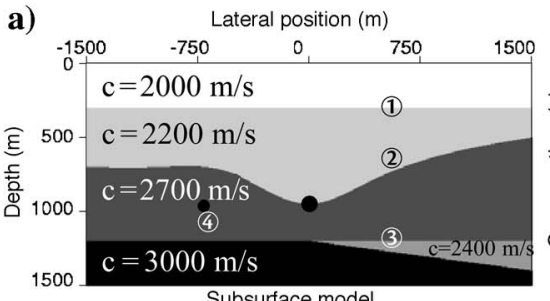

c)

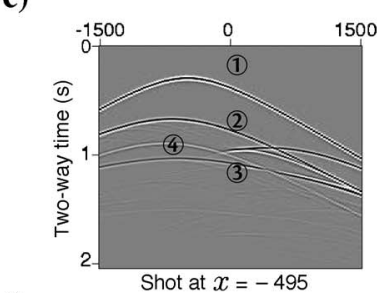

b)

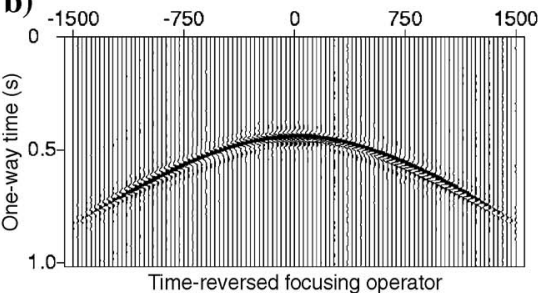

d) e) f)

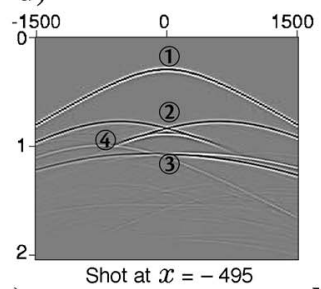

$-1500$

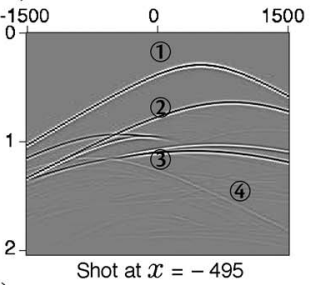

g) $\quad 0 \quad$ h)

h)

Shot at $x=-495$

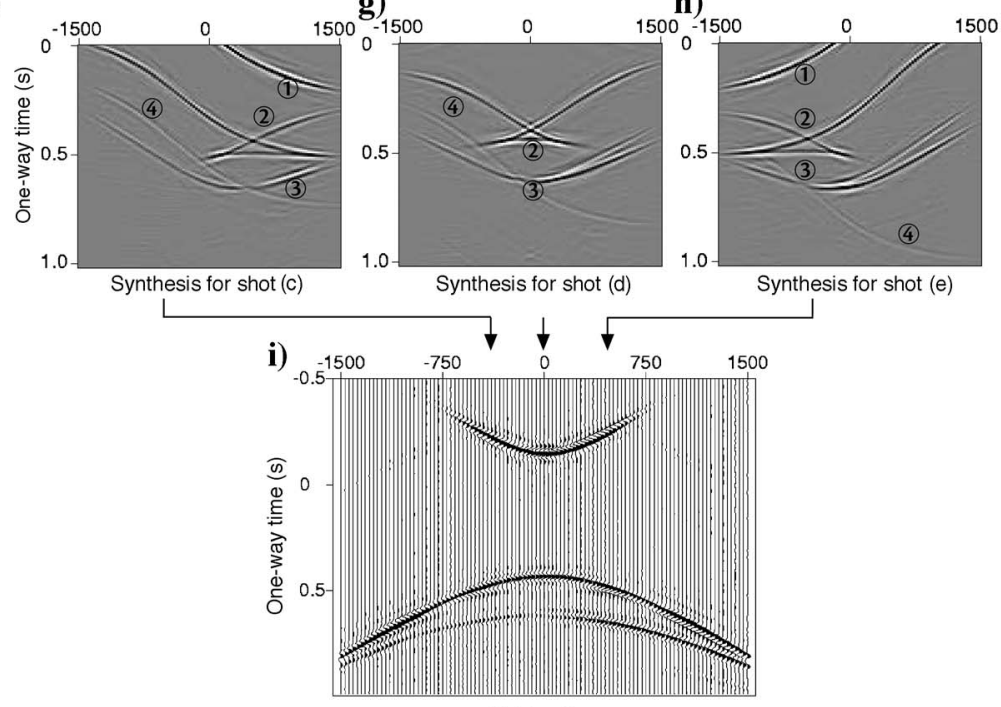

CFP gather

Figure 3. Construction of a CFP gather for focusing in detection. The focus point response has been indicated with an arrow in (i), showing the same traveltime as the operator (principle of equal traveltime) in (b). The focusing process transforms each shot record in (c), (d), and (f) into one trace that is positioned in the CFP gather at the source position of the corresponding shot record. The horizontal parts in (f), (g), and (h) (Fresnel zones) contribute to the result in (i). Note that the CFP gather can be seen as a shot record with an areal source given by the focusing operator. Note also that the CFP gather does not contain multiarrival times: The spatial phase has been removed, leading to simpler events than in the original shot record (d).

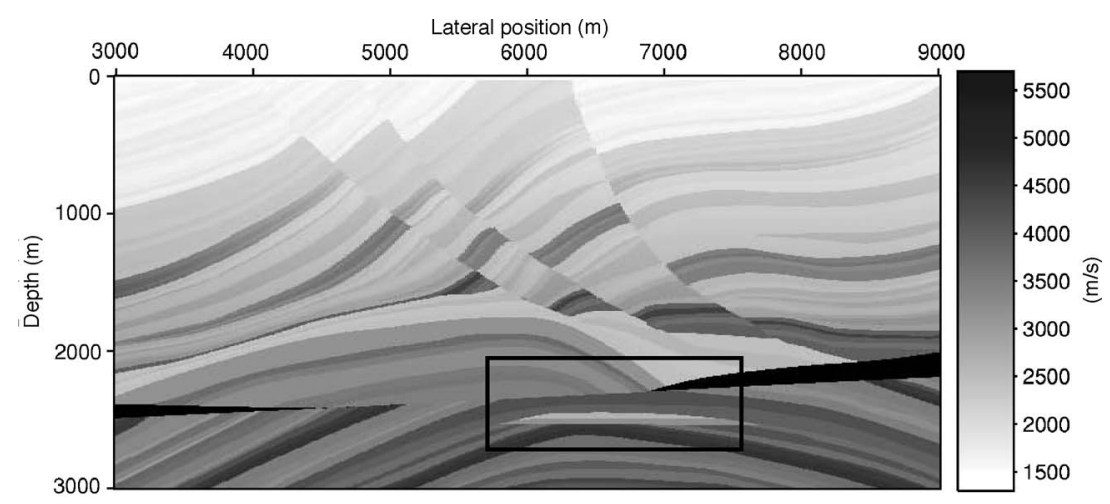

Figure 4 . The velocity model used to model the Marmousi data set. The frame indicates a potential hydrocarbon reservoir. ery CFP gather. To construct these gathers, we utilize the Marmousi data set, whose velocity model is shown in Figure 4.

The Marmousi data of the Institut Français du Pétrole is based on a complex geologic model (Versteeg and Grau, 1991). These data provide a challenge for any migration method, even when using correct velocities. The acquisition geometry is a marine type of acquisition (end of spread) containing 96 geophone groups with a minimum offset of $200 \mathrm{~m}$ and a receiver spacing of $25 \mathrm{~m}$. The modeled data contain 240 shots with a shot spacing of $25 \mathrm{~m}$. A single trace has a length of $4 \mathrm{~s}$ with a sampling interval of $4 \mathrm{~ms}$. The potential hydrocarbon reservoir is positioned around $x$ $=7000 \mathrm{~m}, z=2500 \mathrm{~m}$ and can be recognized by the horizontal levels in the turtleback structure.

The preprocessing for the Marmousi data set was conducted in a two-step approach (Rietveld, 1995) in which the surface-related multiples and the thin-layer reverberations were removed. The missing near offsets were interpolated using a common midpoint (CMP) interpolation technique. This preprocessed data set, including the interpolated offsets, was also used as input for the migration algorithms discussed below.

In Figures 5 and 6, CFP-offset gathers are compared with shot-based offset gathers. The main difference between the two sections is that in shot-based offset gathers, the traces are single fold: Only one trace out of a shot gather is used in the offset gather. In the CFP-based offset gathers, every trace has been constructed using all the traces in a shot record. The CFP-offset gathers therefore have a higher quality and are easier to interpret. By choosing different focal levels, for example, at $z=3000 \mathrm{~m}$ in Figure 6, deeper parts of the model can be interpreted better.

Figure 7 compares shot-record migration with CFP-gather migration. Note the difference in illumination area (compare, in Figure 7, $\mathrm{c}$ with $\mathrm{h}$ and $\mathrm{d}$ with i) when the source is placed at the surface (shot based) or the deep subsurface (CFP based). It can also be seen that by using CFP gathers, a target-oriented approach around the chosen focal points is possible. By using CFP and imaging equations $8-11$, it can be shown that the operator $\mathbf{F}_{j}\left(z_{s}, z_{f}\right)$ used to construct the CFP gather cancels out in the final imaging result. This means that velocity errors in $\mathbf{F}_{j}\left(z_{s}, z_{f}\right)$ have no influence on the image gather. Thus, one can choose any kind of focusing operator as long as the source and receiver gathers used in the migration use the same focusing operator.

\section{CFP GATHER MIGRATION EXPERIMENTS}

Several imaging experiments have been carried out to test and compare the image quality of 
CFP-gather migration with conventional migration of shot records in the presence of noise. For the migration and the construction of the focusing operators, different velocity models have been used to illustrate the influence of velocity errors.

\section{Results on the 2D Marmousi model}

Figure 8 shows six conventional shot-record migration results. For the left-hand side, pictures of all available shots are used, and for
Figure 5. Comparison between shot- and CFPoffset (focus depth $z_{f}=300 \mathrm{~m}$ ) gathers. The CFP-offset gather can be considered a redatumed data set where the receiver positions have been inverse extrapolated to $z=300 \mathrm{~m}$. Near the sea bottom, the CFP-based offset gather has better quality than the shot-based offset gather.

Figure 6. Comparison between shot- and CFPoffset (focus depth $z_{f}=3000 \mathrm{~m}$ ) gathers. The CFP-based offset gather gives a better image of the deeper subsurface than shot-based offset gathers. This favorable property is used in the CFP-based image gathers.

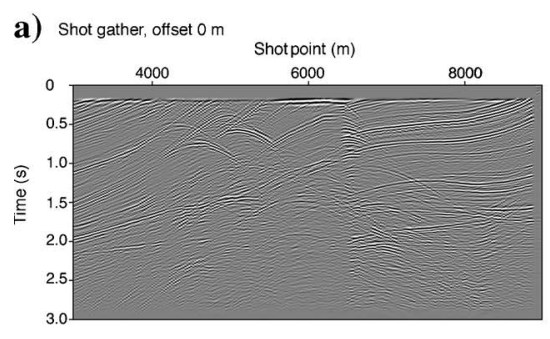

b) CFP gather, $z_{f}=300 \mathrm{~m}$, offset $0 \mathrm{~m}$
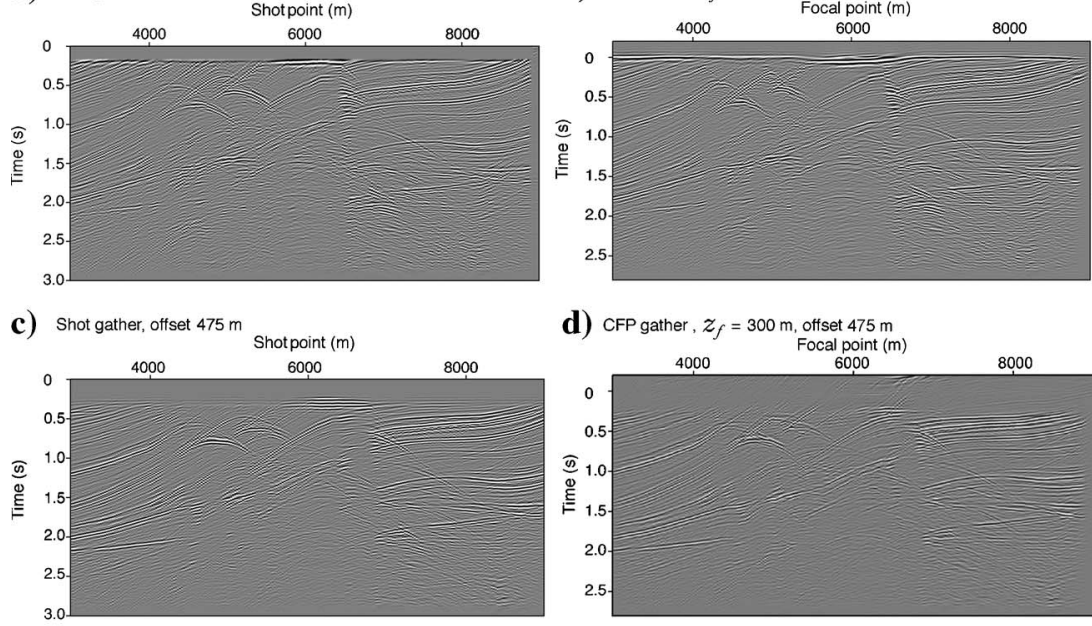

d) CFP gather, $z_{f}=300 \mathrm{~m}$, offset $475 \mathrm{~m}$

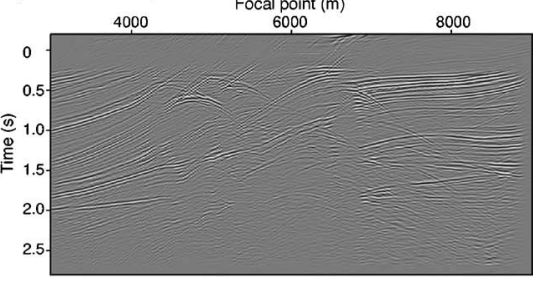

e) Shot gather, offset $1300 \mathrm{~m}$ Shot point (m)

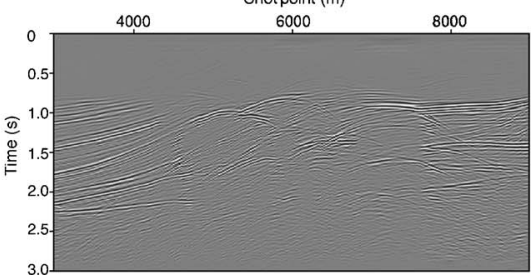

f) CFP gather, $z_{f}=\begin{array}{r}300 \mathrm{~m} \text {, offset } 1300 \mathrm{~m} \\ \text { Focal point }(\mathrm{m})\end{array}$

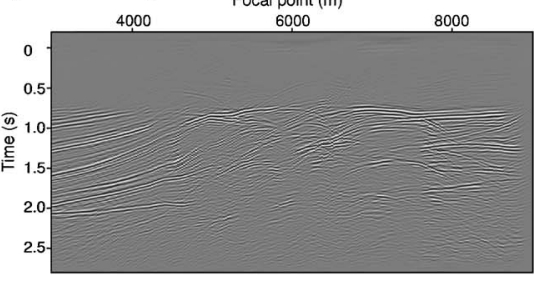

a) Shot gather, offset $0 \mathrm{~m}$

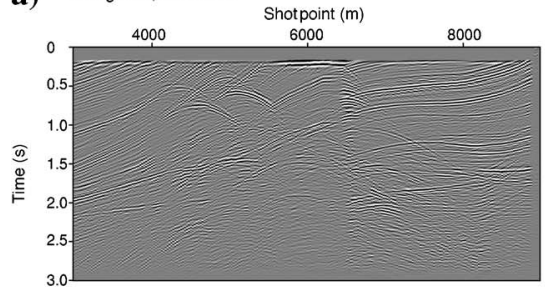

b) CFP gather, $z_{f}=3000 \mathrm{~m}$, offset $0 \mathrm{~m}$

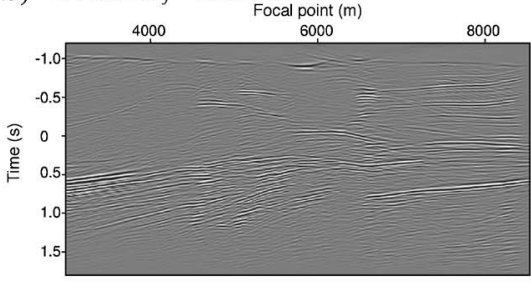

c) Shot gather, offset $475 \mathrm{~m}$ Shot point (m)

d) CFP gather, $z_{f}=3000 \mathrm{~m}$, offset $475 \mathrm{~m}$
Focal point (m)
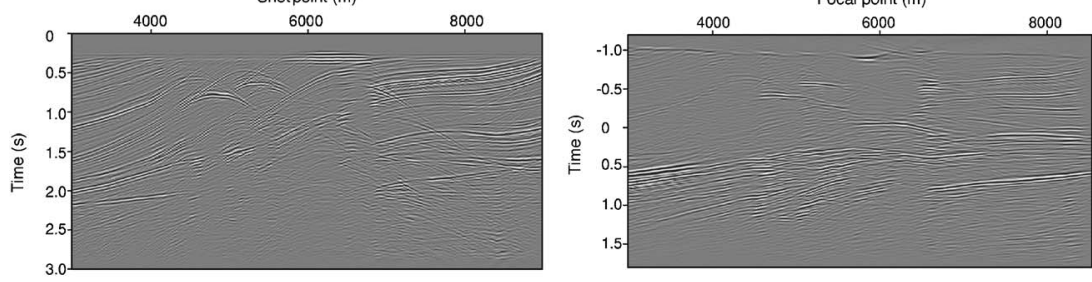

e) Shot gather, offset $1300 \mathrm{~m}$

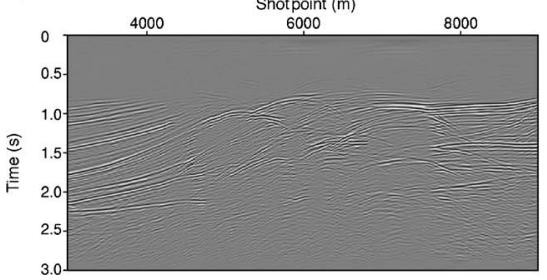

f) CFP gather, $\boldsymbol{z}_{f}=3000 \mathrm{~m}$, offset $1300 \mathrm{~m}$

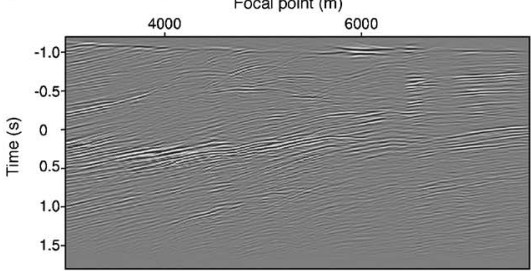


Figure 7. Comparison between a migrated-shot record (top) and a migrated CFP gather (bottom). (a) A shot record and (b) its source wavefield. In CFP gather migration, the shot record (a) is replaced by the CFP gather (e), and the source (b) is replaced by the focusing operator (f). In this example, the focal point of the CFP gather is chosen at $z_{f}=3000 \mathrm{~m}$. Note the large difference in illumination between the image of shot-record migration in (c) and CFP gather migration in (h).

Figure 8. Conventional shot-record migration using different shot-sampling intervals $\left(\Delta x_{s}\right)$, and different $\mathrm{S} / \mathrm{N}$ ratios. For the migration, the correct velocity model has been used. The left figures are based on all 240 shots; the right pictures are based on 60 shots. Views (a) and (b) are comparable in image quality; (e) and (f) are clearly different. Because of the use of all shot records, (e) has a better reduction of noise than (f).
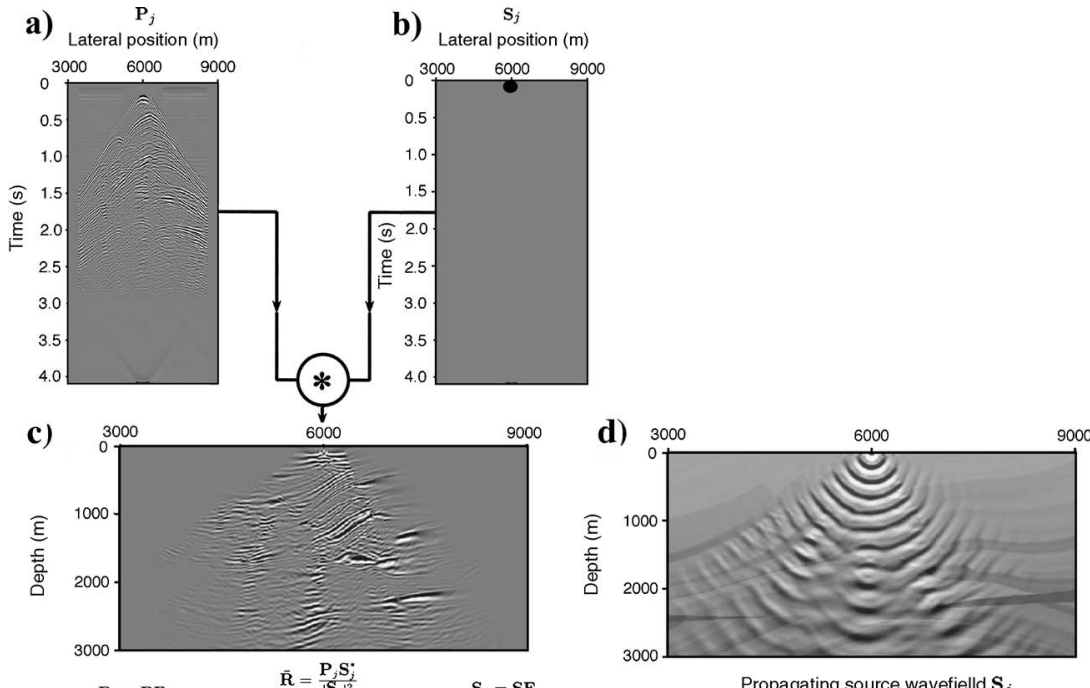

$\begin{aligned} \mathbf{P}_{j} & =\mathbf{P F}_{j} \\ \text { e) Lateral position (m) } & \end{aligned}$
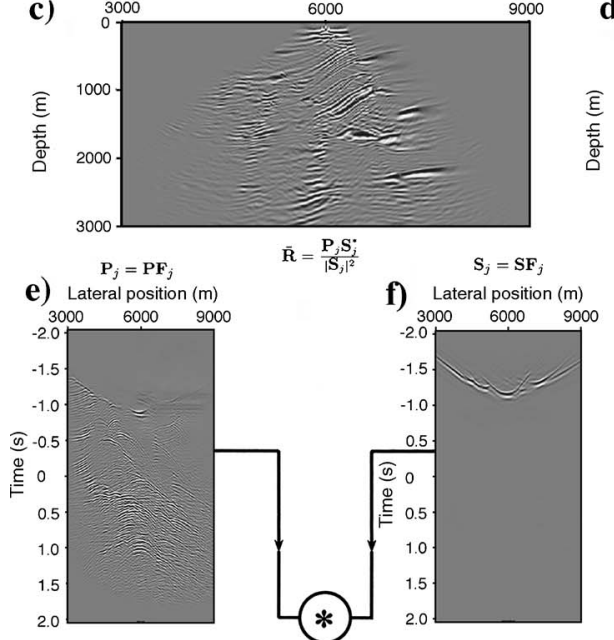

h)

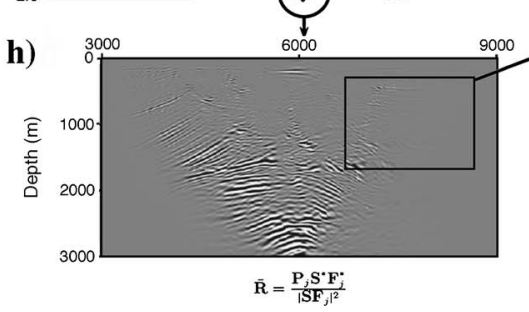

a) $\Delta x_{s}=25 \mathrm{~m}$ (240 shot records)

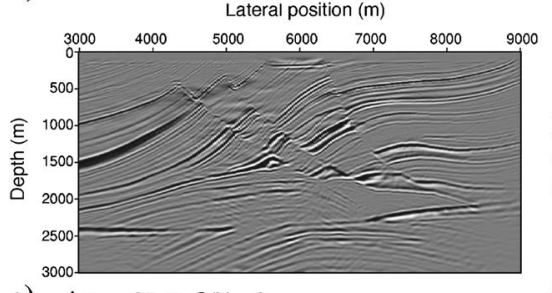

c) $\Delta x_{s}=25 \mathrm{~m}, \mathrm{~S} / \mathrm{N}=2$

1) $3000 \quad 6000 \quad 9000$

Propagating source wavefielld $\mathbf{S}_{j}$

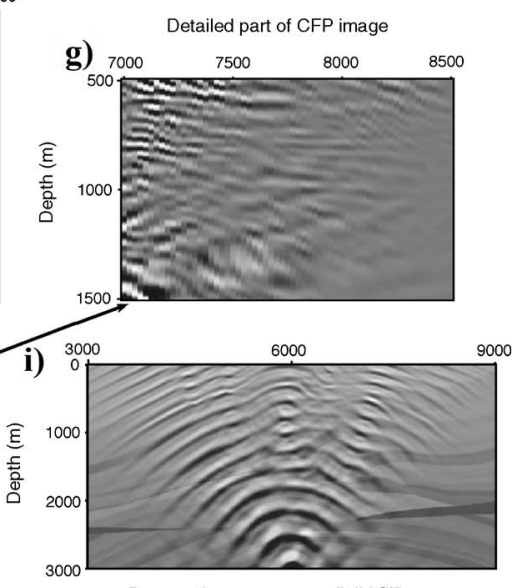

Propagating source wavefielld $\mathbf{S} \mathbf{F}_{j}$

b) $\Delta x_{s}=100 \mathrm{~m}$ (60 shot records)

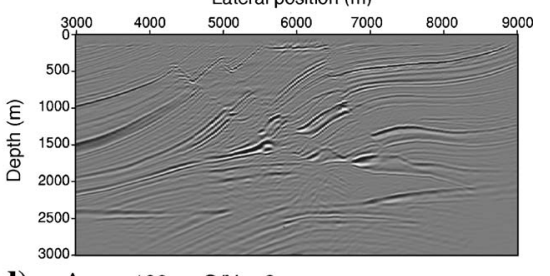

d) $\Delta x_{s}=100 \mathrm{~m}, \mathrm{~S} / \mathrm{N}=2$
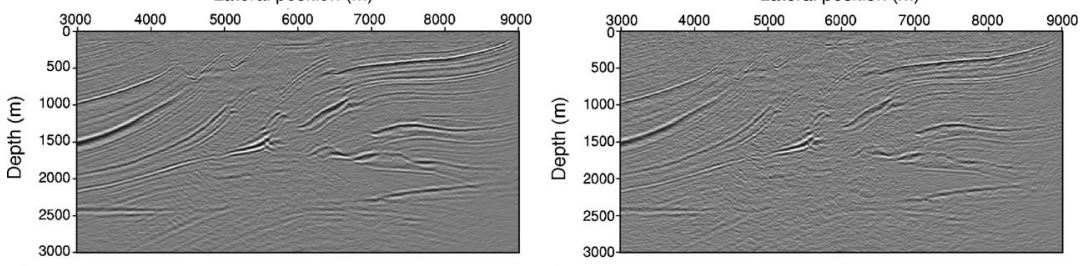

e) $\Delta x_{s}=25 \mathrm{~m}, \mathrm{~S} / \mathrm{N}=1$

f) $\Delta x_{s}=100 \mathrm{~m}, \mathrm{~S} / \mathrm{N}=1$
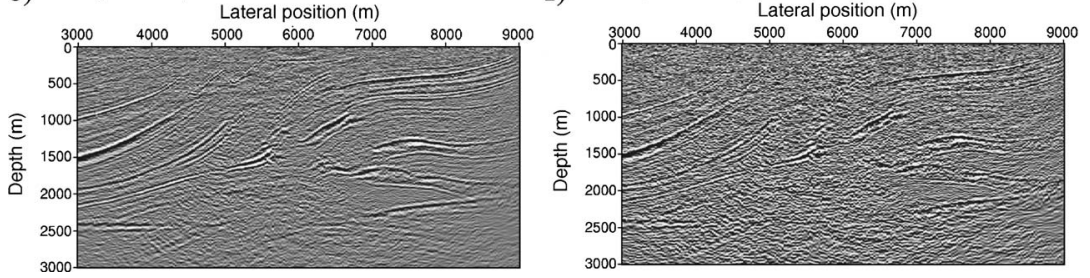
the right-hand side, only one-fourth of the total shots are used. For Figure $8 \mathrm{c}$ and $\mathrm{d}$, Gaussian noise is added to the input shot records with a signal-to-noise $(\mathrm{S} / \mathrm{N})$ ratio of two, and for Figure $8 \mathrm{e}$ and $\mathrm{f}$, with a ratio of one. Figure 8 a shows the full prestack-migration result using all 240 shots of the Marmousi data set $\left(\Delta x_{s}=25 \mathrm{~m}\right)$. Figure $8 \mathrm{~b}$ shows the image for $\Delta x_{s}=100 \mathrm{~m}$. The difference in image quality between Figure 8a and b can hardly be observed, but the computation time for Figure $8 \mathrm{~b}$ is four times less. For the results based on the noisy data, the effect of using fewer shot records is visible in a lower $\mathrm{S} / \mathrm{N}$ ratio in the migration result. Compared to Figure $8 \mathrm{e}$, Figure $8 \mathrm{f}$ has a much poorer image quality.

Figure 9 shows the results for CFP-gather migration. The focal points are chosen at a depth of $z=3000 \mathrm{~m}$, with different lateral distances between the focal points at that depth. For the left-hand-side images, 240 focal points are used $\left(\Delta x_{f}=25 \mathrm{~m}\right)$; for the right-handside images, 60 focal points only $\left(\Delta x_{f}=100 \mathrm{~m}\right)$. The migrations in Figures 8 and 9 are carried out with different noise levels added to the input-shot records. Comparing the noise-added CFP-gather migration results of Figures 9 with the noise-added shot-record-migration results in Figure 8, the CFP-gather migration has a much better $\mathrm{S} / \mathrm{N}$ ratio because the noise has been reduced already by Fresnelzone stacking. The closer we approach the focal depth boundary (here $z_{f}=3000 \mathrm{~m}$ ), the more effective this property is.

Related to these observations, one could design an operator so that all Fresnel zones for a certain focal area in the true model (whose exact position is not relevant) are present in the CFP gather. The Fresnel zones present then are not summed up to an optimal stack, but at least they have a nonvanishing contribution in the CFP gather and hence this energy can be migrated. An example of such an opera- tor is an operator modeled in a very smooth velocity model. The smoothed velocity model has been made so that no multiarrivals occur in the one-way wavefields. The effect of using this operator in the construction of a CFP gather is shown in Figure 10. The top part, representing shot-record migration, shows that using this smoothed model breaks down the shot migration result; many unmigrated events observed as smiles are still present. The CFP operator $\left(\mathbf{S}_{j}\right.$ $=\mathbf{S F}_{j}$, Figure 10f calculated in this smoothed model looks very simple compared to the one in Figure 7f. For the migration of the CFP gather, the true model has to be used again to calculate the correct image. As stated before, as long as the CFP gather and its focal source are consistent, they may be based on a velocity model that is different from the true model. Compared to Figure 7h, the CFPmigrated image in Figure 10h shows more imaged reflectors (for example look at the area around $x=8000 \mathrm{~m}, z=1000 \mathrm{~m}$ and compare the zoomed-in part (g) of the CFP image). Using this smooth focusing operator, the CFP gather contains Fresnel zones of more reflectors. In addition, the smooth focusing operator does not have one focal point but, rather, a focal area.

The result of CFP-gather migration with operators modeled in a smooth velocity model is shown in Figure 11. For these experiments, the CFP gathers are constructed with an operator modeled in a smoothed velocity model, but the CFP-gather migration is carried out with the correct model.

An important difference between shot-record and CFP-gather migration can be observed in image gathers, constructed from migrated shot records and migrated CFP gathers. The image gathers for the CFP-gather migration are constructed in a way similar to the way the image gathers build up from shots: Every migrated CFP gather contributes to the image gather at a chosen lateral position.
Figure 9. CFP-gather migration using different focal-point sampling intervals $\left(\Delta x_{f}\right)$ at $z=3000$ $\mathrm{m}$, and different $\mathrm{S} / \mathrm{N}$ ratios. For the focusing operators, the correct velocity model has been used. In contrast with Figure 8, (e) and (f) have comparable quality. Using CFP, the noise reduction has already taken place in the focusing step by Fresnel-zone stacking; migration of fewer CFP gathers still gives a good $\mathrm{S} / \mathrm{N}$ ratio.
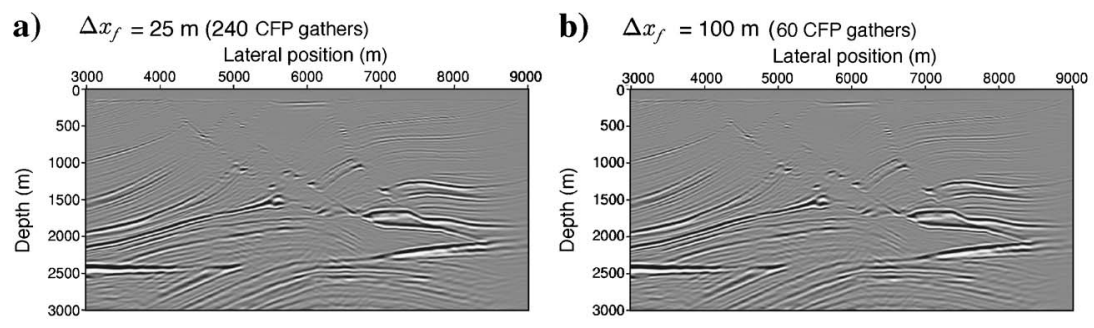

c) $\Delta x_{f}=25 \mathrm{~m}, \mathrm{~S} / \mathrm{N}=2$

d) $\Delta x_{f}=100 \mathrm{~m}, \mathrm{~S} / \mathrm{N}=2$

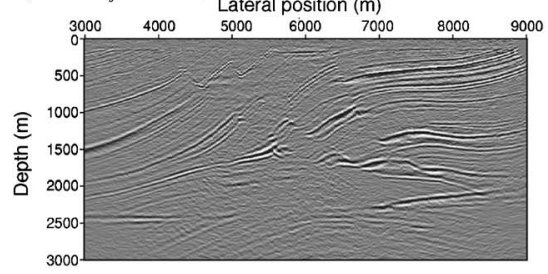

d) Lateral position $(\mathrm{m})$

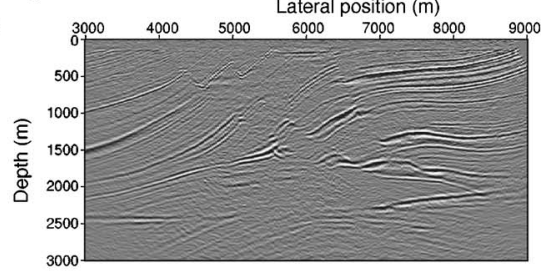

e) $\Delta x_{f}=25 \mathrm{~m}, \mathrm{~S} / \mathrm{N}=1$

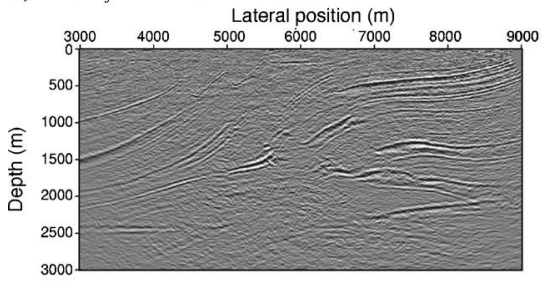

f) $\Delta x_{f}=100 \mathrm{~m}, \mathrm{~S} / \mathrm{N}=1$

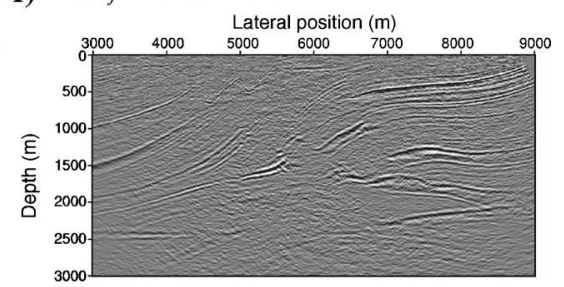


a) $\stackrel{\mathbf{P}_{j}}{\text { Lateral position (m) }}$

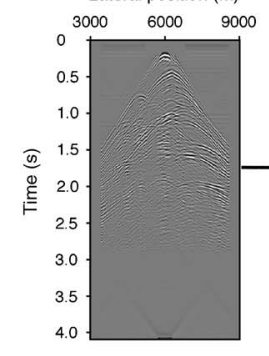

c)
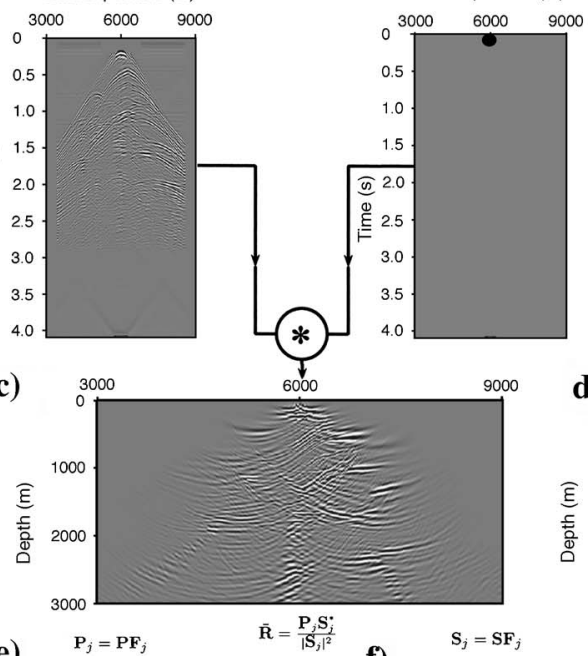

b) $\stackrel{\mathrm{S}_{j}}{\text { Lateral position (m) }}$

d)

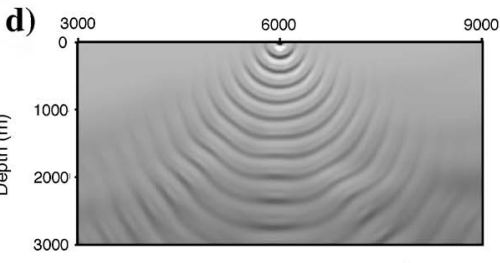

Propagating source wavefielld $\mathbf{S}_{j}$

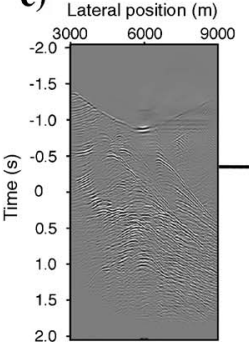

h)

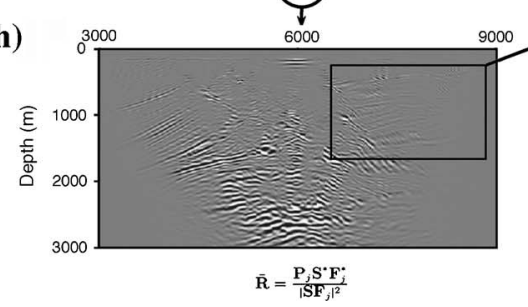

a) $\Delta x_{f}=25 \mathrm{~m}$ (240 CFP gathers)

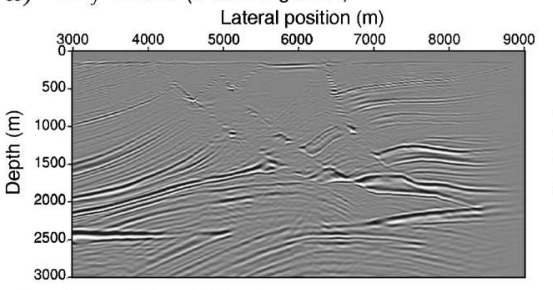

c) $\Delta x_{f}=25 \mathrm{~m}, \mathrm{~S} / \mathrm{N}=2$

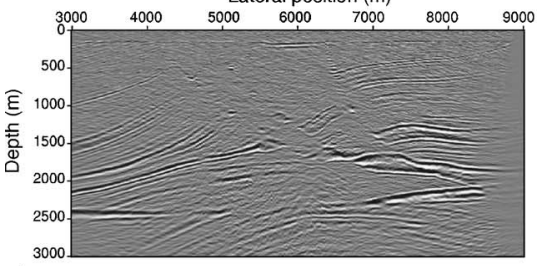

e) $\Delta x_{f}=25 \mathrm{~m}, \mathrm{~S} / \mathrm{N}=1$

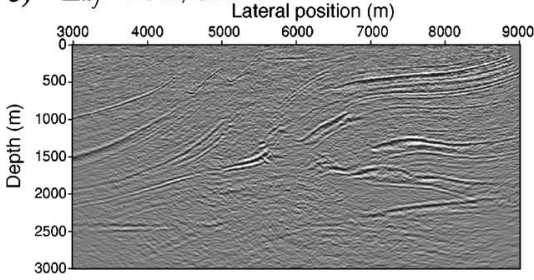

b) $\Delta x_{f}=100 \mathrm{~m}$ (60 CFP gathers)

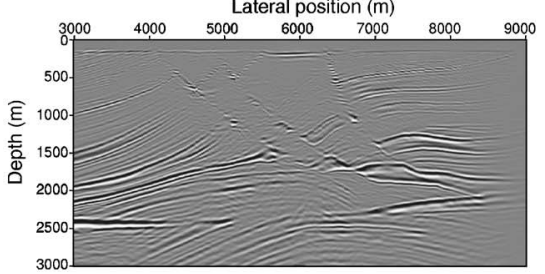

d) $\Delta x_{f}=100 \mathrm{~m}, \mathrm{~S} / \mathrm{N}=2$ Lateral position (m)

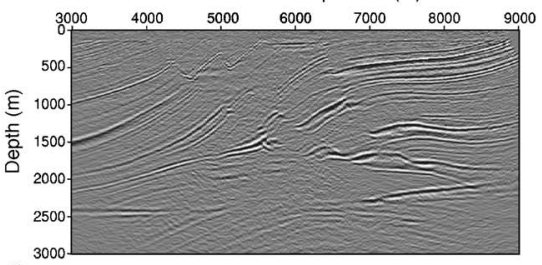

f) $\Delta x_{f}=100 \mathrm{~m}, \mathrm{~S} / \mathrm{N}=1$ Lateral position $(\mathrm{m})$

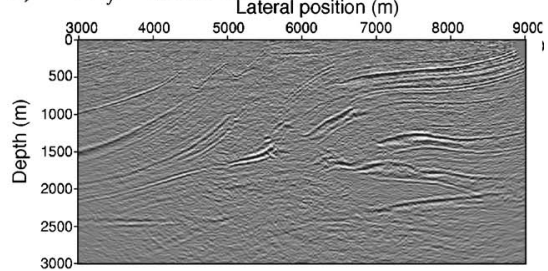

Figure 10. Migration carried out with an averaged ( $300 \times 300 \mathrm{~m}^{2}$ smoothing operator) velocity model. (c) The image of the shot-record migration contains many unmigrated events observed as smiles. In the migration of CFP gathers, a different velocity model is used for the focal operator (f) and in imaging (h). For the computation of the focal operator, the smooth model was used. For the CFP-gather migration, the true model was used. In the imaging step, the focal operator is canceled out.
Figure 11. Migration of CFP gathers using different focal-point sampling intervals $\left(\Delta x_{f}\right)$ at $z=3000 \mathrm{~m}$ and different $\mathrm{S} / \mathrm{N}$ ratios. The velocity model to generate the focal operators and the CFP gathers has been smoothed $\left(300 \times 300 \mathrm{~m}^{2}\right.$ smoothing operator). For the actual migration, the unsmoothed model is used. 
a) Image gathers without noise
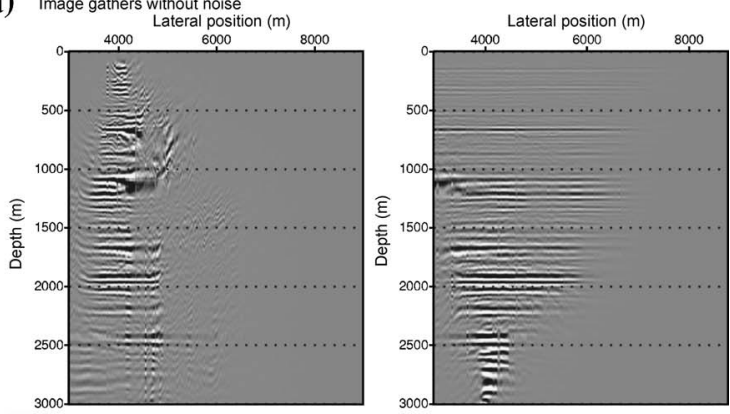

b) Image gathers with $\mathrm{S} / \mathrm{N}=2$
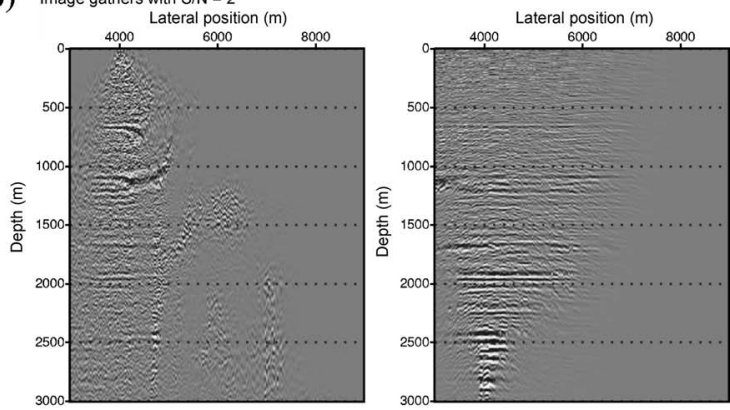

c)
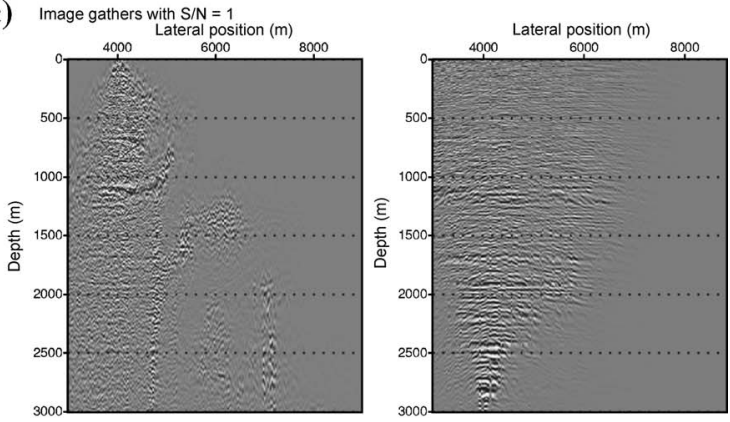

Figure 12. Image gathers for shot-record migration (left panels) and CFP-gather migration (right panels) at $x=4000 \mathrm{~m}$ for different noise levels. The focal depth for the CFP gathers is $z_{f}=3000 \mathrm{~m}$. As expected, the CFP-based image gathers have better continuity of the events and the $\mathrm{S} / \mathrm{N}$ ratio is higher. The correct model has been used to calculate the focal operators and to carry out the migration.

The image gathers of Figures 12 and 13 show that the CFP-image gathers (right-hand side) have a better lateral continuity compared to the shot-record image gathers (left-hand side). Near the focal point of the CFP gathers, at $z=3000 \mathrm{~m}$ in Figure 12 and $z=300 \mathrm{~m}$ in Figure 13, the lateral continuity shrinks because of the focusing of the source energy. The $\mathrm{S} / \mathrm{N}$ ratio in the image gather from CFP gathers (pictures $b$ and $c$ in Figures 12 and 13) is higher and therefore better suited for velocity analysis. The summation over the Fresnel zone reduces the spatially incoherent noise. To reduce the coherent noise, extra Fresnel-zone filters could be applied. Note that in the CFPbased image gathers source-receiver reciprocity is considered.

The reflectors that are far from the focal point in the CFP-image gather will have a $\mathrm{S} / \mathrm{N}$ ratio comparable to that of shot-record migration. This is because at the focal point, the Fresnel zone stack is opti- a) Image gathers without noise
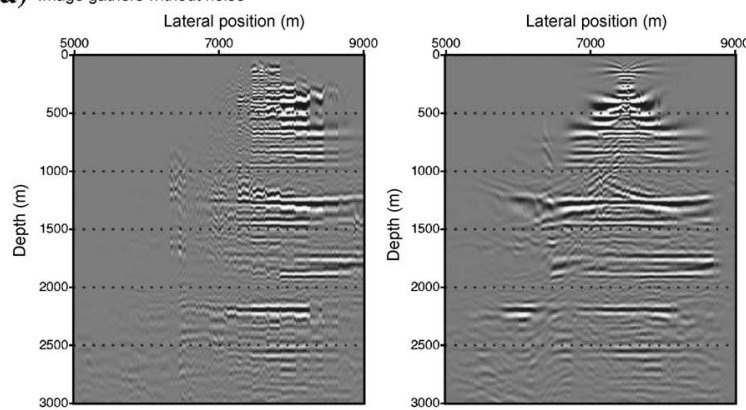

b) Image gathers with $S / N=2$
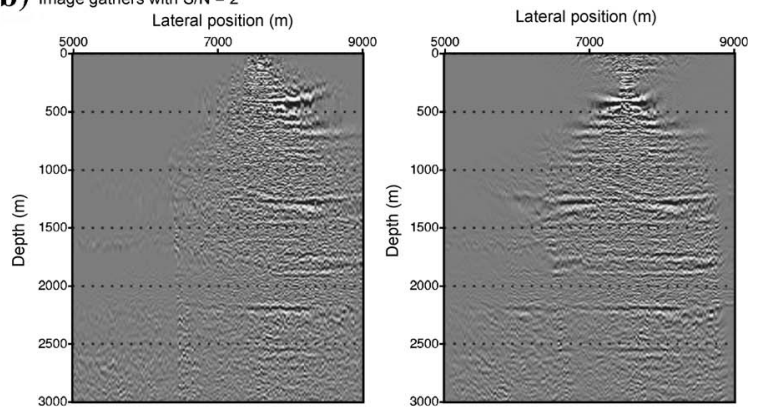

c) Image gathers with $S / N=1$
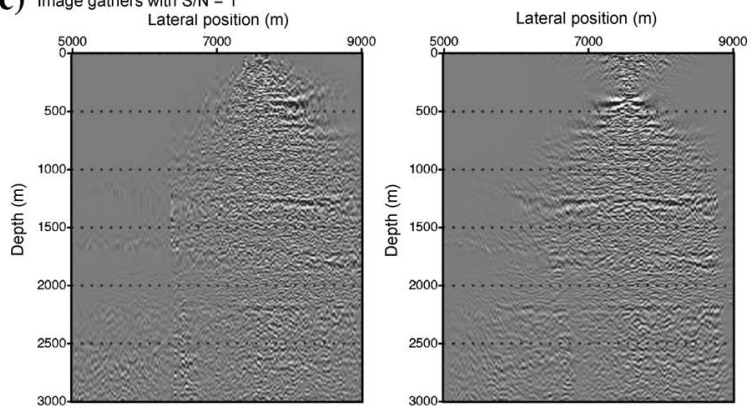

Figure 13. Image gathers for shot-record migration (left panels) and CFP-gather migration (right panels) at $x=7500 \mathrm{~m}$ for different noise levels. The focal depth for the CFP gathers is $z_{f}=300 \mathrm{~m}$. The CFP-based image gathers have better continuity of events and the $\mathrm{S} / \mathrm{N}$ ratio is higher. The correct model has been used to calculate the focal operators and to carry out the migration.

mal (will have its largest lateral extent). For all other points, this zone will be smaller, and consequently a smaller improvement of the $\mathrm{S} / \mathrm{N}$ ratio.

CFP-image gathers can also be used for velocity analysis. Figures 14 and 15 show image gathers where an erroneous velocity model has been used. The CFP gather shows the velocity errors more pronounced, and errors can be followed at a larger lateral distance. For the deeper events the shot-based image gathers in Figure 15 show discontinuities caused by the shallower parts in the model. In the CFP-based image gathers, these discontinuities are absent because the shallow subsurface already was accounted for by the focusing operator. By choosing different focal depths for the CFP-image gathers - at $z=3000 \mathrm{~m}$ in Figure 14 and $z=300 \mathrm{~m}$ in Figure $15-$ different parts of the model can be analyzed in more detail. 
a) Image gathers correct model
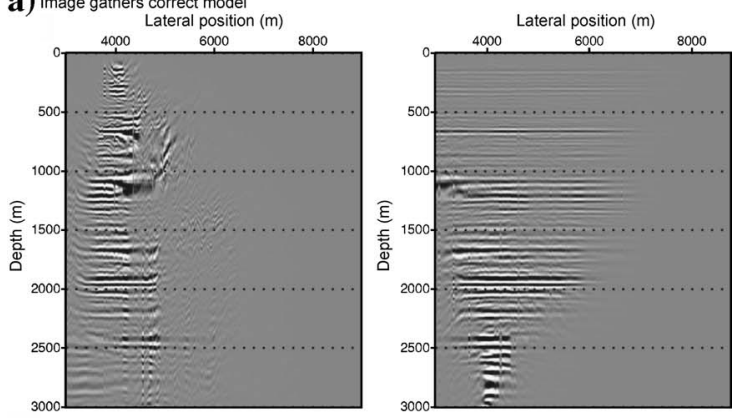

b) Image gathers smooth model

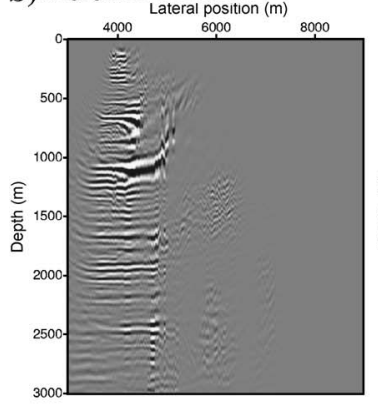

c) Image gathers erroneous model ( $-10 \%)$

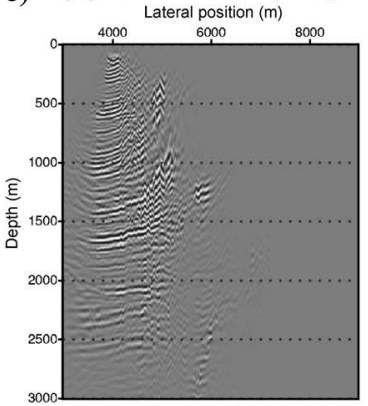

Lateral position (m)
6000
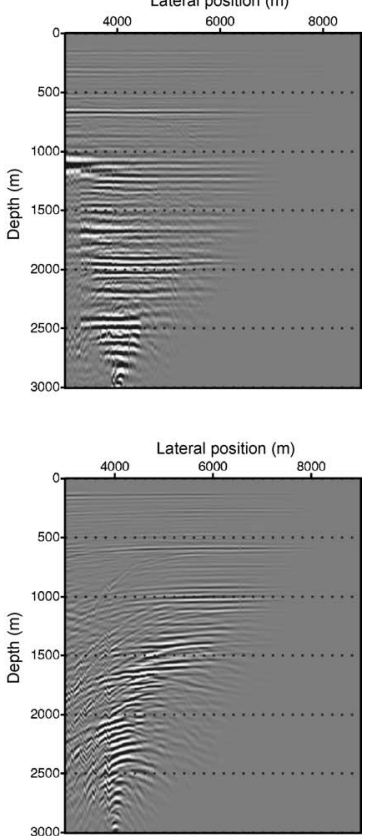

Figure 14. Image gathers for shot-record migration (left panels) and CFP-gather migration (right panels) at $x=4000 \mathrm{~m}$. The focal depth for the CFP gathers is $z_{f}=3000 \mathrm{~m}$. A smoothed version of the velocity model has been used in (b), and an erroneous macro velocity model ( $-10 \%$ for all velocities) in (c). The smooth and erroneous models have been used to calculate the focal operators and to carry out the migration.

\section{CONCLUSIONS}

CFP gathers are constructed from all available shot records and are related to a focus in the subsurface. CFP gathers can be considered as shot records generated by a focal source array and can be migrated with a shot-record migration algorithm.

Focusing involves Fresnel zone stacking. Therefore, CFP gathers have a higher $\mathrm{S} / \mathrm{N}$ ratio than shot records. Because focusing removes spatial phase from the data, CFP gathers contain simpler events than the original shot record.

In a CFP image gather, being based on migrated CFP gathers, each migrated trace contains multifold data. The result is easier to interpret than image gathers based on single-fold migrated field records. Focal operators can be generated in a reference medium that may be different from the medium used in the migration process.

a) Image gathers correct model
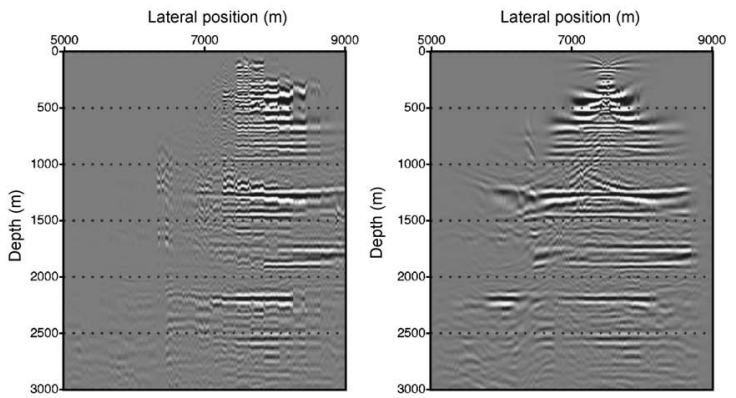

b) Image gathers smooth model
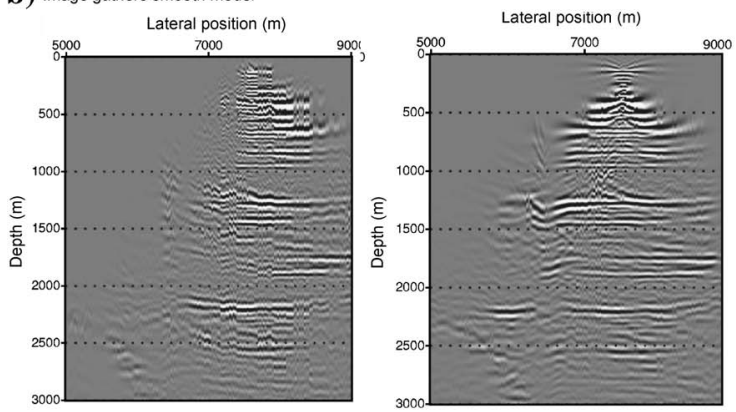

c) Image gathers erroneous model ( $-10 \%)$
Lateral position (m)
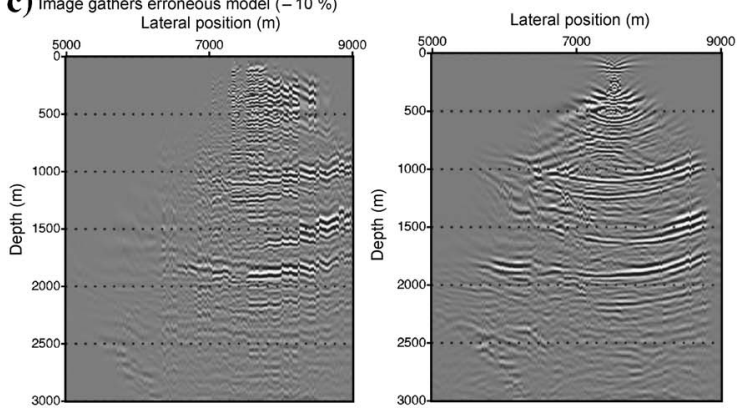

Figure 15. Image gathers for shot-record migration (left panels) and CFP-gather migration (right panels) at $x=7500 \mathrm{~m}$. The focal depth for the CFP gathers is $z_{f}=300 \mathrm{~m}$. A smoothed version of the velocity model has been used in (b), and an erroneous macro velocity model ( $-10 \%$ for all velocities) in (c). The smooth and erroneous models have been used to calculate the focal operators and to carry out the migration.

\section{ACKNOWLEDGMENTS}

Jan Thorbecke dedicates this paper to his late wife, Rosalinde Thorbecke-Lagendijk, for her continuous moral support in finishing this paper. He also thanks Cray Inc. for permitting time to work on this research. Both authors would like to thank the Delphi Consortium members for stimulating discussions and financial support.

\section{REFERENCES}

Berkhout, A. J., 1980, Imaging of acoustic energy by wave field extrapolation: Elsevier Science Publishing Company, Inc.

-, 1982, Imaging of acoustic energy by wave field extrapolation, 2nd ed.: Elsevier Science Publishing Company, Inc.

1992, Areal shot record technology: Journal of Seismic Exploration, 1, 251-264.

, 1997, Pushing the limits of seismic imaging, Part I: Prestack migration in terms of double dynamic focusing: Geophysics, 62, 937-953. Berkhout, A. J., and D. J. Verschuur, 2000, Internal multiple removal - 
Boundary-related and layer-related approach: 62nd Annual International Meeting, EAGE, Extended Abstracts, L-56.

Blacquière, G., 1989, 3D wave field extrapolation in seismic depth migration: Ph.D. thesis, Delft University of Technology.

Bolte, J., D. Verschuur, and R. Hegge, 1999, CFP operator estimation and inversion demonstrated on a field data set, Part I: Operator updating: 69th Annual International Meeting, SEG, Expanded Abstracts, 1711-1714.

Brisbourne, A., T. Pointer, and H. Crook, 2000, Improved imaging beneath complex near-surface structure using CFP velocity analysis: 62nd Annual International Meeting, EAGE, Extended Abstracts, L-34.

Hegge, R., A. Duijndam, J. Bolte, and J. Fokkema, 1999, CFP operator estimation and inversion demonstrated on a field data set, Part II: Velocity estimation: 69th Annual International Meeting, SEG, Expanded Abstracts, $1500-1503$.

Holberg, O., 1988, Towards optimum one-way wave propagation: Geophysical Prospecting, 36, 99-114
Kabir, M. M. N., and D. J. Verschuur, 1997, Velocity analysis of the complex subsurface using the common focus point technology: 67th Annual International Meeting, SEG, Expanded Abstracts, 1822-1825.

Kelamis, P., D. Verschuur, A. Berkhout, and K. Erickson, 1999, Velocity-independent datuming of seismic data: 69th Annual International Meeting, SEG, Expanded Abstracts, 441-444.

Rietveld, W. E. A., 1995, Controlled illumination in prestack seismic migration: $\mathrm{Ph} . \mathrm{D}$. thesis, Delft University of Technology.

Thorbecke, J., 1997, Common focus point technology: Ph.D. thesis, Technical University of Delft.

Thorbecke, J., K. Wapenaar, and G. Swinnen, 2004, Design of one way wavefield extrapolation operators, using smooth functions in WLSQ optimization: Geophysics, 69, 1037-1045.

Versteeg, R., and G. Grau, 1991, The Marmousi experience: Proceedings of the 53rd Annual International Meeting, Workshop on Practical Aspects of Seismic Data Inversion, EAGE, 1-194. 